and ammonium chloride in sealed tubes, whereby a mixture of chlorides in nearly theoretical amount is obtained. This is distilled from the open tube and afterwards submitted to careful fractional distillation in vacuo. The unique feature of the series is found in the fact that any member can be converted into the rubber-like polymer by heating, and that the latter, on distilling at a higher temperature, breaks down quantitatively into a mixture of all the lower members ; it is, therefore, possible ultimately to convert any member completely into any other by heating and distilling alone. The lower members, up to and including $\left(\mathrm{PNCl}_{2}\right)_{6}$ are well characterized, finely crystallized bodies, while $\left(\mathrm{PNCl}_{2}\right)_{7}$ is liquid. Their stability diminishes with increasing molecular weight, $\left(\mathrm{PNCl}_{2}\right)_{3}$ being unattached by boiling alkalies, while the rubber-like polymer is destroyed by boiling water. The formation of $\mathrm{PNCl}_{2}$ and $\left(\mathrm{PNCl}_{2}\right)_{2}$ could not be detected. The chloronitrides constitute the first extended series of inorganic polymers interconvertible by simple and direct means.

Dr. Fireman gave an extended résumé of the work of Duclaux and others showing that the lactic acid producing bacteria are not so important in the production of butter flavors as are the peptonizing bacteria.

Drs. de Schweinitz and Dorset stated that in studying the products of the growth of the tuberculosis bacilli in artificial media, it was noted that the reaction of the cultures usually becomes acid, and as Prudden and Hodenpyl had succeeded in producing tuberculous nodules without necrosis by the intravenous injection of dead bacilli, it seemed as though it should be possible to isolate from cultures of tuberculosis bacilli an acid substance, which is responsible for the necrosis of tissue that always takes place in this disease. After many fruitless attempts they succeeded in isolating from artificial cultures a crystalline acid substance having a melting point of $161^{\circ}$ to $164^{\circ} \mathrm{C}$. which was soluble in ether, alcohol and water, and crystallized in needle-like prisms. The solution of this substance was optically inactive and did not give the biuret action. The preliminary analysis of this substance gave a formula, like that of teraconic acid and the other properties correspond closely with this acid. Its identity if such has not yet been proved. When injected subeutaneously into guinea pigs it causes a slight inflammation and localized necrosis, and injected directly into the liver tissue by means of a hypodermic syringe it produces characteristic necrosis. The substance causes a reduction of temperature in tuberculous animals, and it seems probable that we have here the material which is responsible for the necrosis in tubercular infection.

Dr. Wiley and Mr. Krug showed that various methods depending on the polarization of the starch or its inversion products, as well as others based on direct weighing of the starch, were too inaccurate to commend themselves to the analyst. The only accurate methods depend on the eventual inversion of the starch into dextrose, which is then estimated by Allihn's method. Experiments in the preparation of the Nitro-naphthalenes showed that they can not be prepared from $a$-naphthalene sulphonic acid, but that the best yield is obtained with mixtures of nitric and sulphuric acids. The best results were obtained with $30^{\circ}$ B. nitric acid, using about three times as much acid as naphthalene. The amount of sulphuric acid, used varies with the nitration degree desired, varying from $4: 1$ (nitric: sulphuric) for low melting products to $3: 2$ for the higher derivations.

Dr. Cameron cited some of his own experiments to show that, although it is possible in the case of some heavy metals to replace chlorine by sulphur by merely boiling the latter with a solution of the chloride, it is impossible to obtain such a reaction with alkaline, or alkaline earth chlorides even upon heating in sealed tubes at a high temperature.

Mr. Tassin gave a short description of a new mineral, but reserves the details for special publication.

\section{K. Chesnut.} Secretary.

TORREY BOTANICAL CLUB, MAY 26, 1887.

The President, Hon. Addison Brown, presided. The evening was devoted to a lecture by Mrs. Elizabeth G. Britton, entitled 'The 
Moss Flora of the Adirondack Mountains,' illustrated by lantern-slides prepared by Mr. C. H. Van Brunt, and also by numerous mounted specimens. These specimens, comprising about 150 sheets, handsomely displayed about the walls of the lecture-room, represented collections made by Mrs. Britton in the vicinity of Adirondack Lodge and Lake Placid in the years 1892, 1894 and 1896. The various locations where these mosses grew were described, including the story of a climb up Mount Whiteface. Graphic bits of description of these mosses brought out salient points as the slides indicating their structure were exhibited, and these were accompanied by numerous slides illustrating the scenery of their habitat. Among about 30 rare species enumerated were Raphidostegium Jamesii, not previously reported for New York State, and Bryum concinnatum, found only once before in the United States. Duplicates of Mrs. Britton's collection have been deposited at the State Herbarium at Albany, the main collection having been presented to the Herbarium of Columbia University. Partial sets were sent to the Brooklyn Institute, to Cornell University and to various other collections.

The subject of the lecture was further discussed by Mr. A. P. Grout and by Mrs. Britton, after whieh the Club adjourned to the second Tuesday in October, field meetings continuing meantime on Saturdays.

\section{Edward S. Burgess, Secretary.}

SCIENCE CLUB OF THE UNIVERSITY OF WISCONSIN.

Mr. Edward Kremer discussed 'The Periodic System.' The speaker introduced his subject by giving a brief account of the earlier attempts to refer back to a prime matter the manifold forms in which material nature reveals herself to man. This line of thought was briefly traced from the early Greek philosophers to Prout. The reaction that followed Prout's hypothesis was duly emphasized. The lack of interest in Doehereiner's trials, and the modifications of these trials by others, in Newland's octaves, in Lothar Meyer's first chart it was thus explained in large part. Attention was also called to the fact that the principal interests seemed to center about the numerical relation existing between the atomic weights of the elements and that the time value of the system was not duly appreciated until this was made secondary to the natural classification of the elements according to their physical and chemical properties. Incidentally the prediction by Mardelejiff of the existence of certain elements and their discovery by others were alluded to, because with the discovery of their elements the natural law gained more general favor. What the periodic law really is was strongly emphasized, also the fact that the present arrangement of the elements according to this law is imperfect, but more recent systems were not discussed, partly for want of time. Finally the great advantage to be gained by using the periodic law as the basis for teaching advanced chemistry was demonstrated

C. S. SLIChter reviewed Larmor's 'Theory of the Ether.' He first gave an explanation of the vortex theory of matter, including the properties of vortex rings and their mode of linking, and kinds of vibration. Hill's spherical vortex was explained by a diagram, and the possibility of building up a rotationally elastic ether from such cells was explained. Photographs of sandstone showing turbulent motion were presented for the purpose of showing the character of their structure. The intrinsic energy of the ether, radiation, action at a distance, density and inertia of common matter were then discussed, closing with a new theory of gravitation. Mr. Quantz gave the results of work on the psychology of rapid reading. They were the results of a statistical study of the mental habits and characteristics of fifty university students, showing that rapidity of reading depends chiefly upon the following factors, given in the order of their importance: (a) quickness of visual perccption; (b) practice, as measured by extent of reading from childhood up ; $(c)$ power of mental concentration; $(d)$ absence of motor-mindedness (lip movement in silent reading); $(e)$ rapidity of mental operations in general; $(f)$ strongly developed eye-mindedness; $(g)$ scholarly ability, as determined by class study.

$$
\text { WM. S. Marshall, }
$$

Secretary. 